\title{
Complement C3 Triggers Malignant Progression of Mesenchymal Subtype Glioma
}

\begin{abstract}
ChunlanNing", YongchangYang", YangXie, YanchengLiu, YidingAi, XingXu, XianglianGe, TailongYi, ShengfuShen, Xun Jin*
\end{abstract}

\begin{abstract}
Department of Biochemistry and Molecular Biology, Tianjin Medical University Cancer Institute and Hospital. National Clinical Research Center for Cancer, Key Laboratory of Cancer Prevention and Therapy, Tianjin. Tianjin's Clinical Research Center for Cancer, Tianjin 300060, China
\end{abstract}

${ }^{\#}$ These authors have contributed equally to this work

*Corresponding author: Xun Jin , Department of Biochemistry and Molecular Biology, Tianjin Medical University Cancer Institute and Hospital. National Clinical Research Center for Cancer, Key Laboratory of Cancer Prevention and Therapy, Tianjin. Tianjin's Clinical Research Center for Cancer, Tianjin 300060, China

\section{Abstract}

Objective: The purpose of this study is to clarify the important role of complement C3 on the chemoradiotherapy resistance of MES subtype glioma.

Methods: We obtained gene expression and clinical data from TCGA, CGGA, Rembrandt and Gravendeel database. We use the LIMMA package to do the differential genes expression analysis. The C3 up-regulation genes did GO and KEGG pathway enrichment analysis. Then, we verify the up-regulation pathway by GSEA. Kaplan-Meier analysis confirmed that C3 also promotes malignant progress in glioma patients after chemoradiotherapy.

Results: The expression of C3 increased with the grade of glioma. C3 was most highly expressed in MES subtype glioma, and mainly enriched in necrotic areas. The C3 up-regulated genes promoted malignant progression of glioma via NF-kB and JAK-STAT signaling pathways. C3 high expression caused chemoradiotherapy resistance, resulting in poor prognosis of glioma patients.

Conclusion: These findings further clarified chemoradiotherapy resistance in MES subtype glioma. In addition, we found that C3 had higher expression in the MES subtype glioma, significantly affecting the prognosis of chemoradiotherapy. We speculated that C3 high expression could cause chemoradiotherapy resistance in glioma. Therefore, targeting C3 and C3 related signaling pathways may provide a new therapeutic approach for chemoradiotherapy resistance to improve the prognosis of glioma patients.

Keywords: complement C3; glioma; mesenchymal subtype; chemoradiotherapy resistance

\section{Introduction}

Glioma is the most common primary intracranial tumor. The World Health Organization (WHO) classification of tumors of the central nervous system is divided into I-IV glioma grade [1]. Among them, the most invasive tumor is glioblastoma (GBM), WHOIV grade, which is characterized by uncontrolled cell proliferation, diffuse infiltration, tendency to necrosis, strong angiogenesis, and chemoradiotherapy resistance $[2,3]$. At the transcriptome level, GBM can be divided into three subtypes: proneural (PN) ,classical (CL) and mesenchymal (MES)[4,5]. However, different subtypes of glioma prefer different tumor microenvironments [6].
Among them, MES subtype glioma is highly enriched in necrosis area with hypoxia and strong inflammation $[7,8]$. Clinically, MES subtype glioma is very difficult to treat, due to its strong chemoradiotherapy resistance $[9,10]$. However, the relationship between chemoradiotherapy resistance and inflammation is unclear. In fact, most inflammation is triggered by a strong immune response [11,12]. As an important part of the innate immune response, complement also plays an important role [13].

Complement has been described as an important factor in the pathogenesis of many central nervous system diseases including infectious, autoimmune and degenerative 


\section{Figure 1}

A

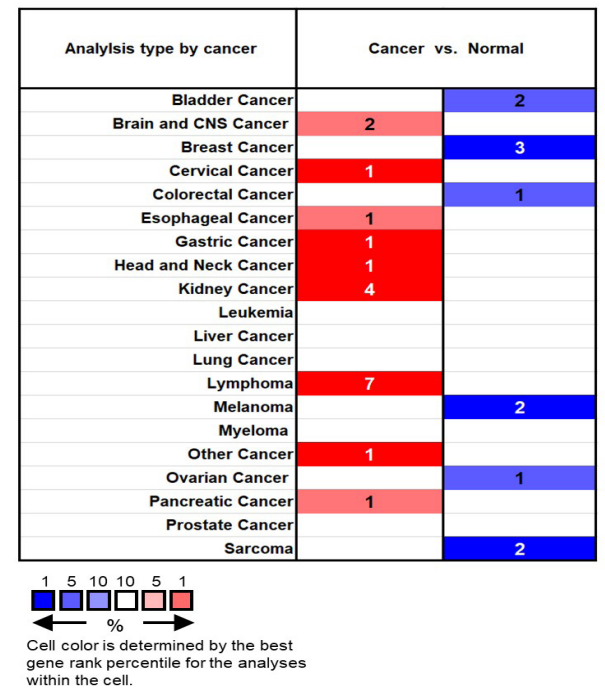

D

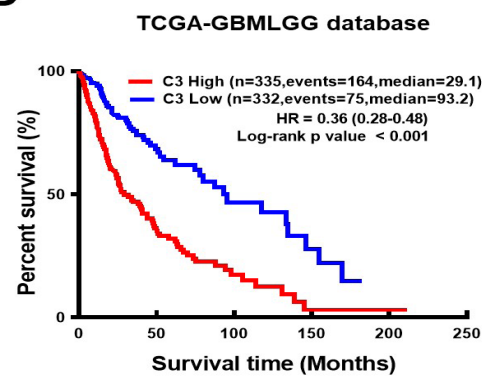

$\mathbf{E}$

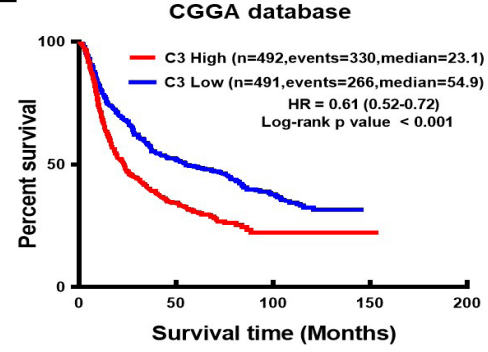

$\mathbf{F}$
B
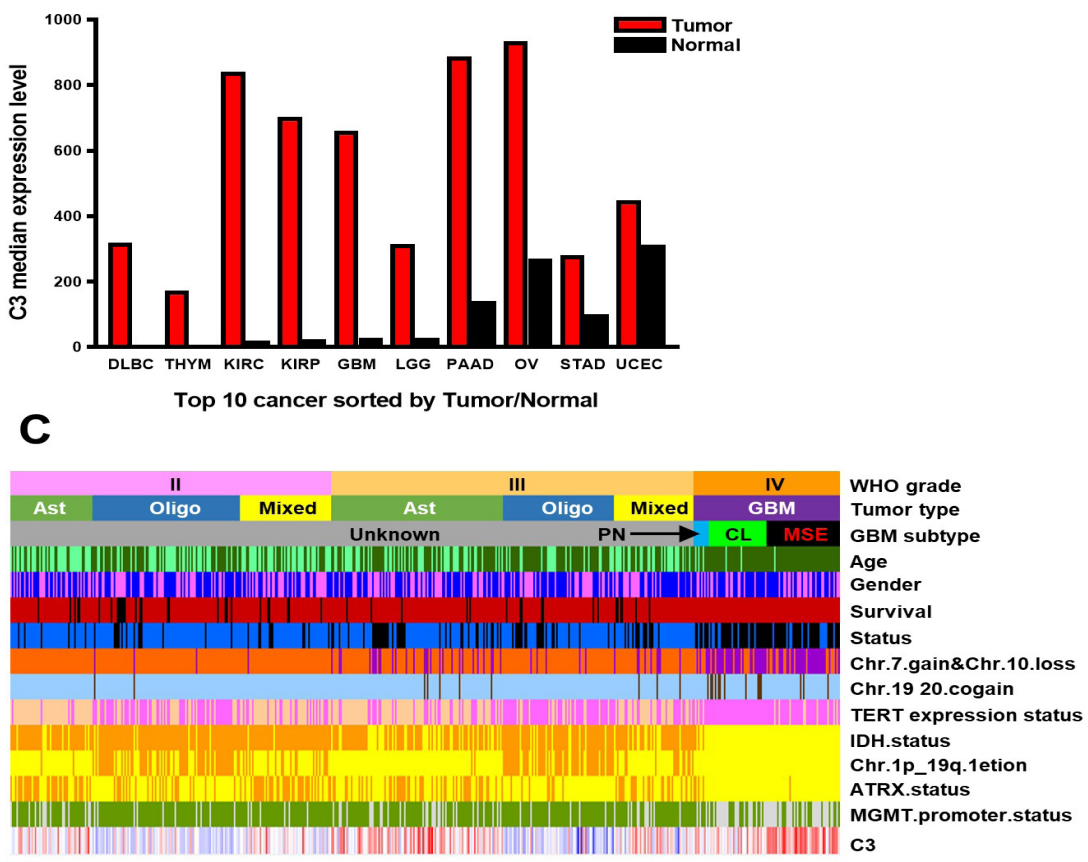

Color legend

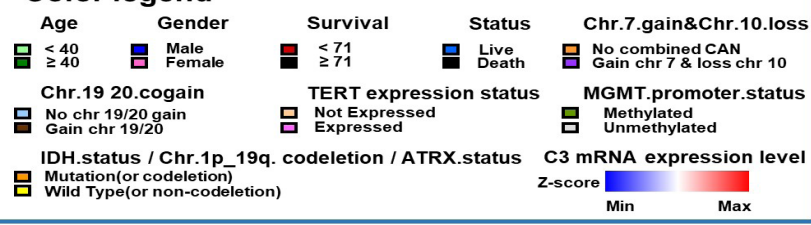

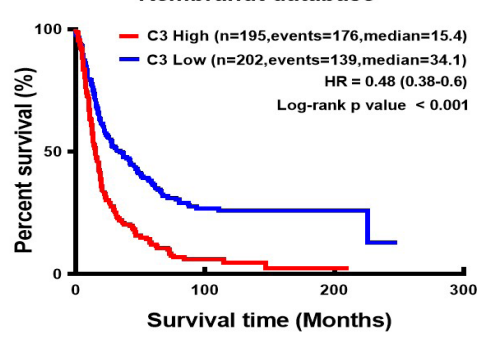

Figure 1: Complement C3 plays an important role in the malignant progression of glioma

(A). The expression of C3 in 20 different types of cancer diseases. Numbers represent the number of high (red) and low (blue) expression databases.

(B). The C3 expression profile across all tumor samples (red) and paired normal tissues (black). The height of bar represents the C3 median expression of certain tumor type or normal tissue.

(C). Heatmap showing distribution of C3 expression and clinical features in TCGA GBMLGG database.

(D-G). Overall survival of GBM patients grouped by C3 median expression in TCGA GBMLGG, CGGA, Rembrandt and Gravendeel database.

Citation: ChunlanNing\#, YongchangYang\#, YangXie, YanchengLiu, YidingAi, XingXu, XianglianGe, TailongYi, ShengfuShen, Xun Jin*.Complement C3 Triggers Malignant Progression of Mesenchymal Subtype Glioma

Op Acc J Bio Sci \& Res 9(1)-2021 


\section{Figure 2}

A
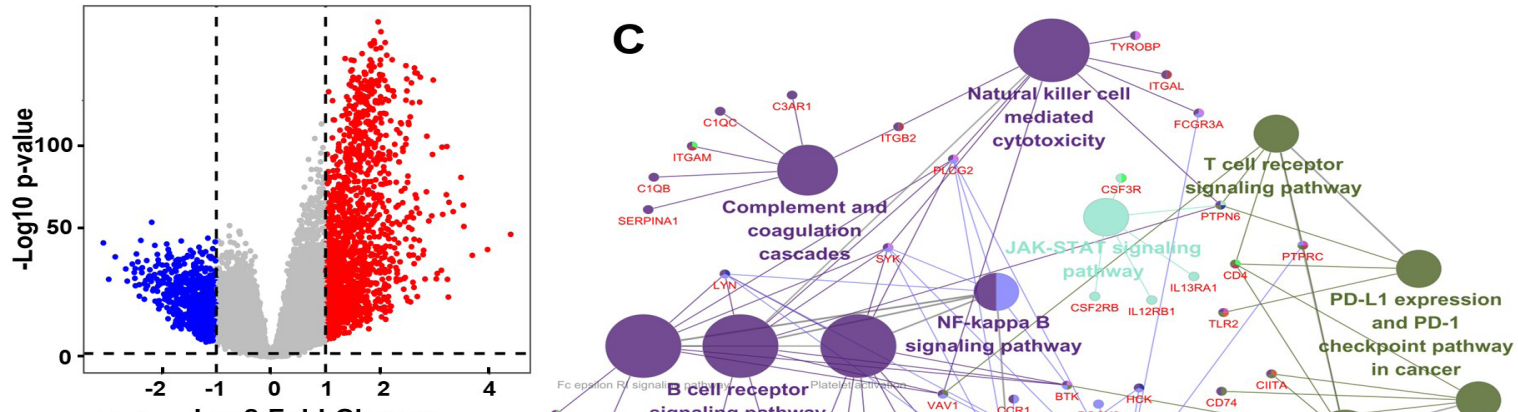

B
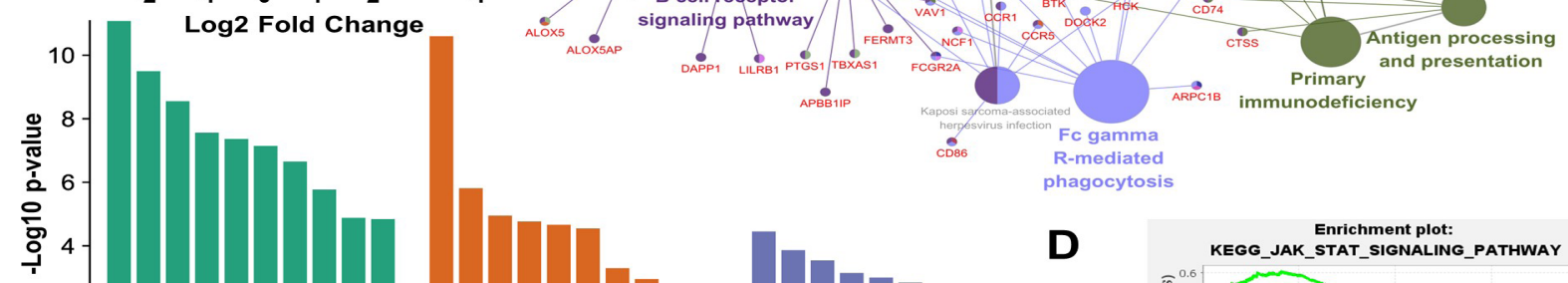

Enrichment plot:

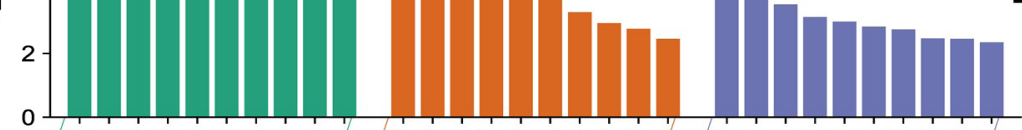

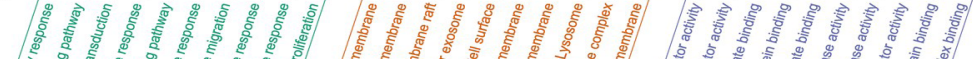

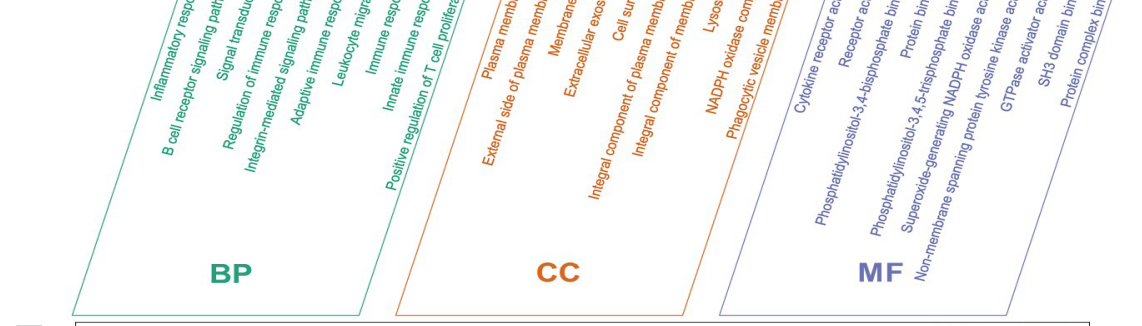

$\mathbf{E}$

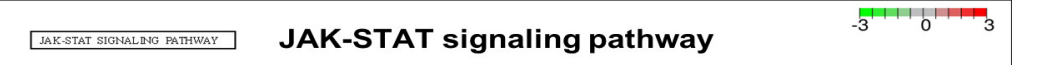

$\mathbf{D}$

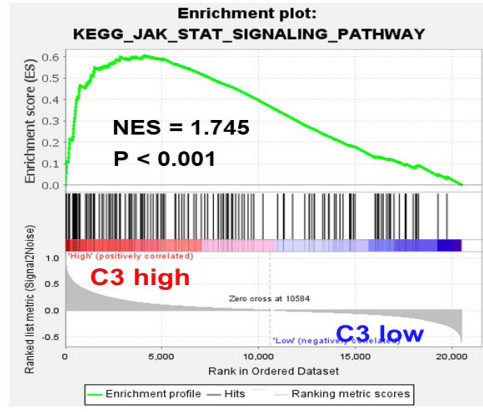

Enrichment plot: KEGG_NFKB_SIGNALING_PATHWAY

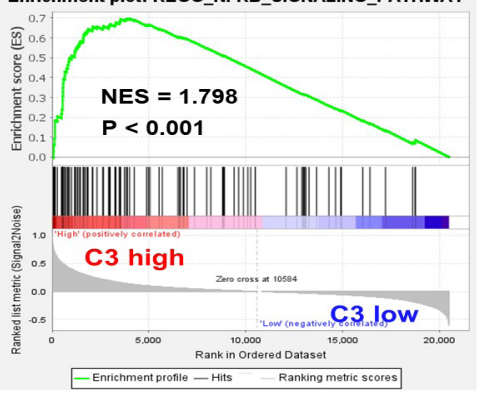

$\mathbf{F}$
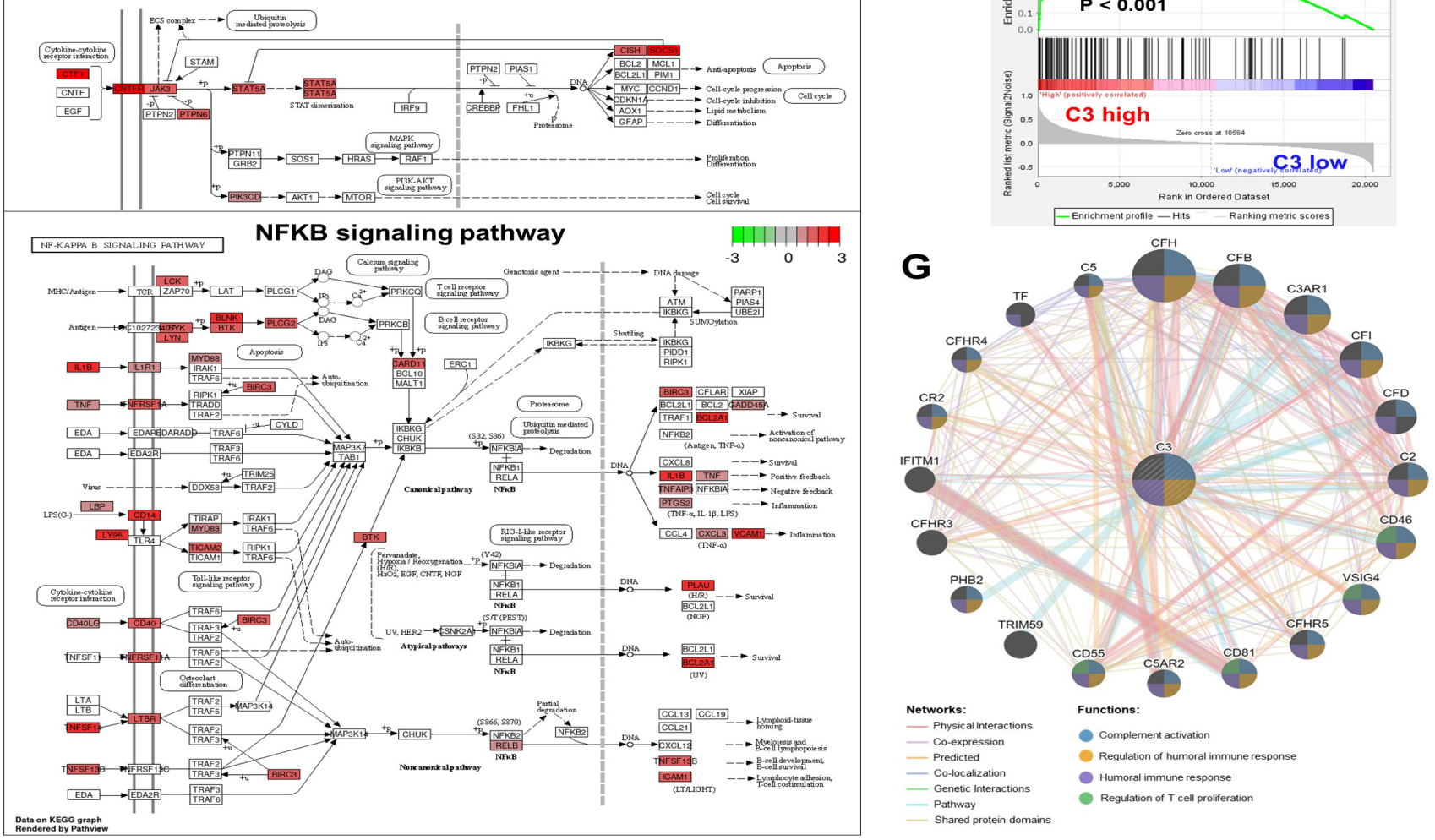

Figure 2: Complement C3 causes malignant progression of glioma through NF-kB and JAK-STAT signaling pathway.

Citation: ChunlanNing\#, YongchangYang", YangXie, YanchengLiu, YidingAi, XingXu, XianglianGe, TailongYi, ShengfuShen, Xun Jin*.Complement C3 Triggers Malignant Progression of Mesenchymal Subtype Glioma

Op Acc J Bio Sci \& Res 9(1)-2021 
(A). Volcano plot for showing C3 differential expression in TCGA GBMLGG database.(fold change $>=2$ and $p<0.05)$. Non-changed genes are shown in gray color. Red color is indicative of up-regulated genes and blue is indicative of down-regulated genes.

(B). Histogram for showing GO biological function enrichment analyses of C3 up-regulated genes. Biological process enrichment analysis (green), Cell component enrichment analysis(orange) and molecular function enrichment analysis(blue).

(C). Network diagram for displaying KEGG pathway enrichment analyses of C3 up-regulated genes. Large circles represent different signaling pathways, and small dots represent genes enriched in pathways. The lines represent the regulation relationship between genes and pathways.

(D). Gene set enrichment analysis (GSEA) between group high C3 expression and C3 low expression shows NFKB and JAK-STAT signaling pathways.

(E) (F). Gene expression in the JAK-STAT signaling pathway and NFkB signaling pathway were plotted by PATHVIEW. Red represents upregulated genes, while green represents down-regulated genes in $C 3$ high expression group.

(G). Construction of gene co-expression networks. The lines represent co-expression networks and the dots represent functions.

\section{Figure 3}

A

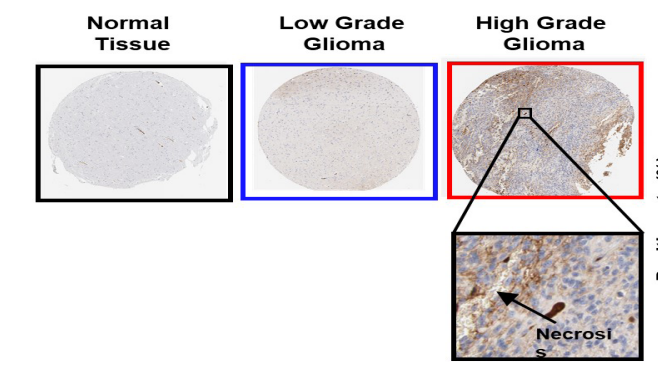

C

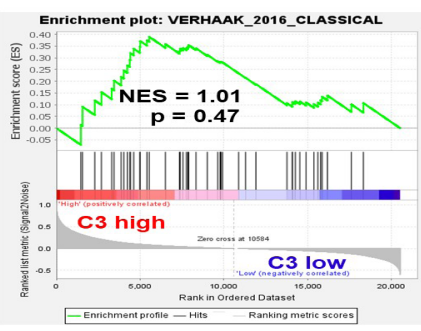

$\mathbf{D}$

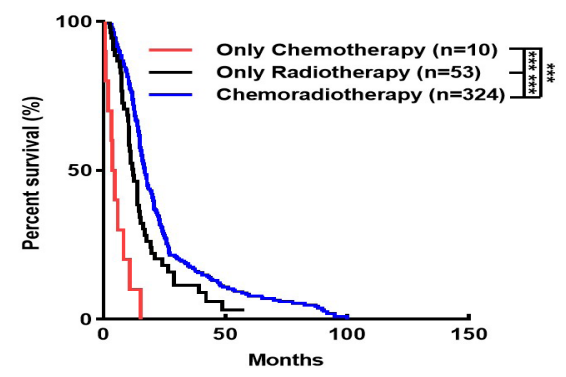

B
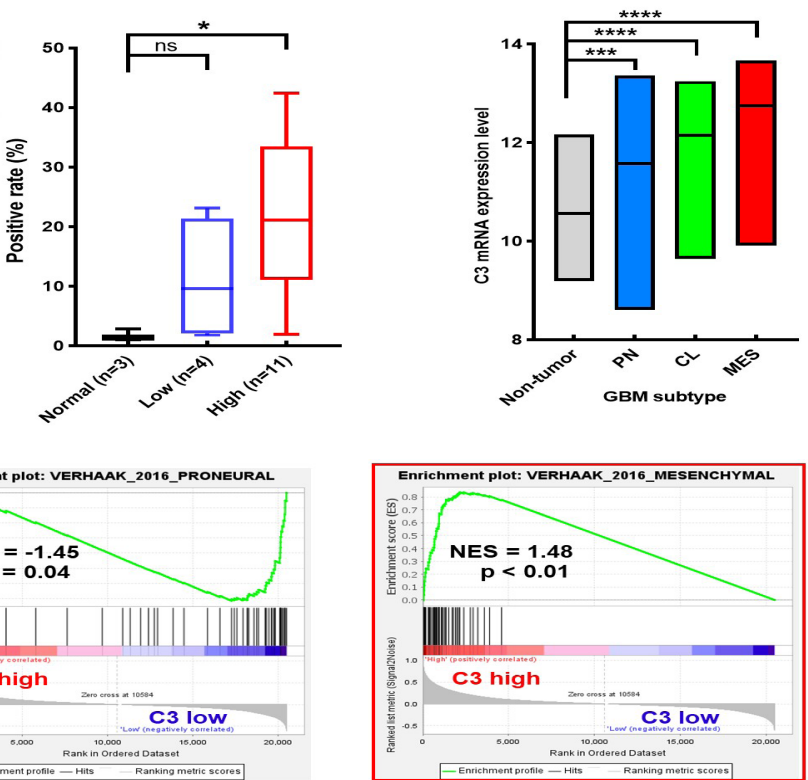

$\mathbf{E}$

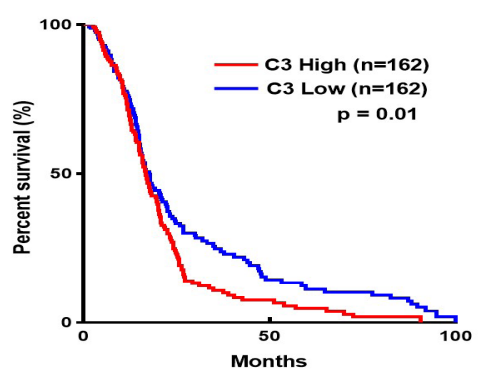

Figure 3: Complement C3 specifically promotes the growth of mesenchymal subtype gliomas and trigger chemoradiotherapy resistance.

(A). The immunohistochemistry of complement $\mathrm{C} 3$ in normal tissues $(n=3)$, low-grade gliomas $(n=4)$, and high-grade gliomas $(n=11)$. The data was downloaded from The Human Protein Atlas. The box plot showing the quantification of the C3 positive rate in different tissues. ' $n s^{\prime}$ represents no significance, ${ }^{*} P<0.05$.

(B). The box plot shows the C3 mRNA expression level of different glioma subtypes in Rembrandt database. (Non-tumor, $n=28 ; P N$, $n=76 ; C L, n=72 ;$ MES, $n=71)$. ${ }^{*} P<0.05, * * P<0.01, * * * P<0.001, * * * * P<0.0001$.

Citation: ChunlanNing\#, YongchangYang", YangXie, YanchengLiu, YidingAi, XingXu, XianglianGe, TailongYi, ShengfuShen, Xun Jin*.Complement C3 Triggers Malignant Progression of Mesenchymal Subtype Glioma

Op Acc J Bio Sci \& Res 9(1)-2021 
(C). Gene set enrichment analysis (GSEA) between group high and low of C3 expression showed signaling pathways of different glioma subtypes in TCGA GBMLGG database.

(D). Kaplan-Meier survival curves for the different treatment groups of the TCGA GBM database (Only Chemotherapy, $n=10 ;$ Only Radiotherapy, $n=53$; Chemoradiotherapy, $n=324)$. ${ }^{*} P<0.05,{ }^{* *} P<0.01,{ }^{* * *} P<0.001$.

(E). Kaplan-Meier survival curves for the high $(n=162)$ and low $(n=162)$ mRNA expression level of C3 in chemoradiotherapy group(n=324).

Table 1: Multivariable Cox Regression Analysis in C3 expression levels and clinical features. ${ }^{*} P<0.05,{ }^{*} P<0.01,{ }^{* * *} P<0.001$.

\section{Hazard ratio}

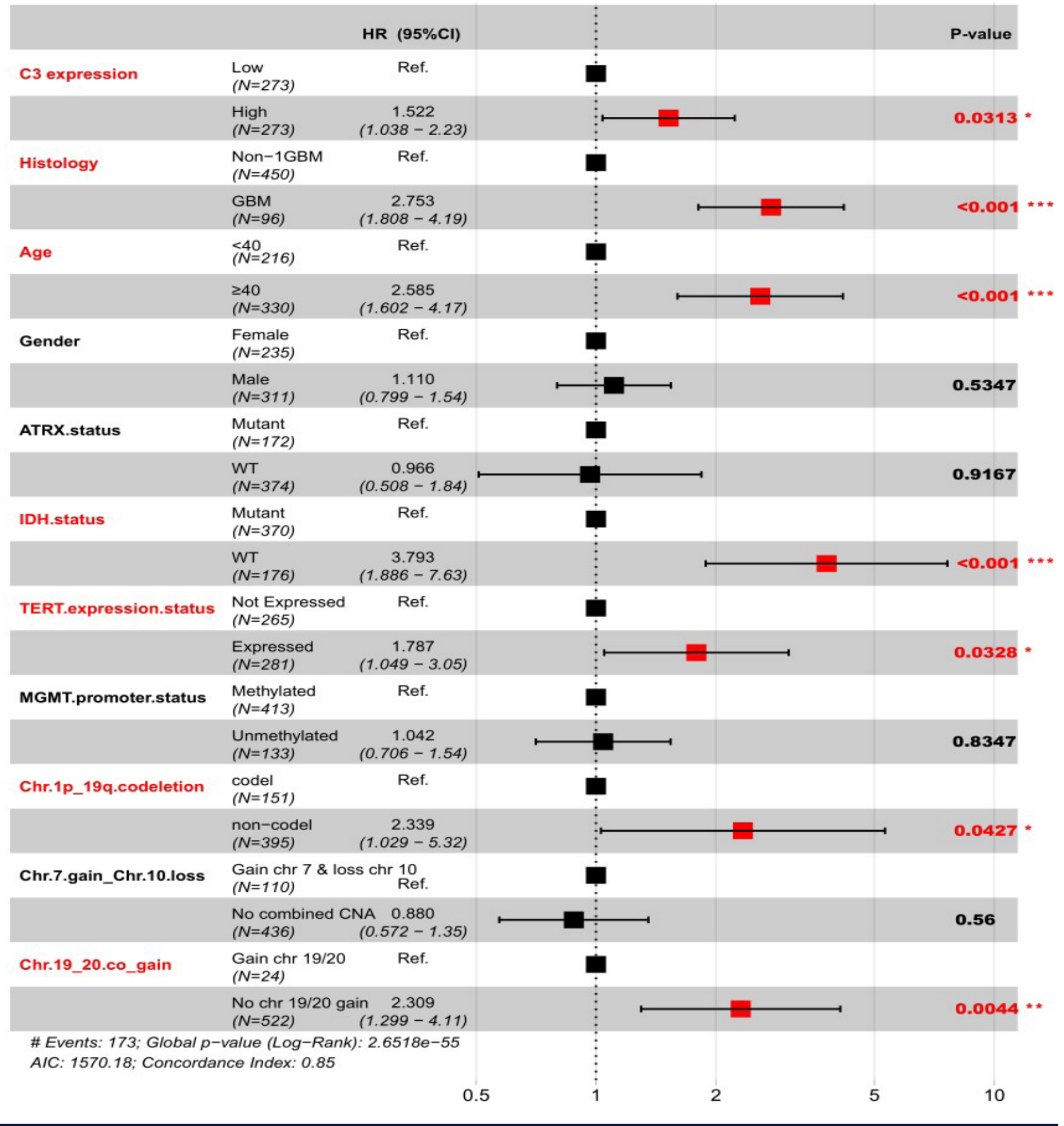

Citation: ChunlanNing\#, YongchangYang\#, YangXie, YanchengLiu, YidingAi, XingXu, XianglianGe, TailongYi, ShengfuShen, Xun Jin*.Complement C3 Triggers Malignant Progression of Mesenchymal Subtype Glioma

Op Acc J Bio Sci \& Res 9(1)-2021 
disorders [14-16]. Complement overexpression is associated with acute brain injury and chronic neurodegenerative diseases including Alzheimer's disease [17, 18] and Huntington's disease [19]. Furthermore, C3 serves as a stage-biomarker of Alzheimer's disease in cerebrospinal fluid (CSF) [20]. Recently, complement C3 was found to be upregulated in all models of meningeal metastasis, and proved to be essential for the growth of cancer in meningea [21]. However, The role of complement C3 in glioma is not certain. In this study, we firstly found that complement C3 is associated with poor prognosis in glioma patients. Then, C3 may regulate malignant progression of glioma through NF-kB and JAK-STAT signaling pathways. Finally, C3 is highly expressed in MES subtype glioma and enhances its chemoradiotherapy resistance.

\section{Material and Methods}

\section{Data Mining from Public Databases}

First, we searched ONCOMINE databases (https:// www.oncomine.org/resource/main.html) to observe the expression of $\mathrm{C} 3$ in different tumors. We searched an online website Gene Expression Profiling Interactive Analysis (GEPIA) (http://gepia.cancer-pku.cn/index.html) to investigate the differential expression of C3 mRNA in glioma tissues and normal tissues. Then, we downloaded the clinical and transcriptional data of glioma patients from TCGA, CGGA, Rembrandt and Gravendeel database (http:// gliovis.bioinfo.cnio.es/). The immunohistochemistry data was downloaded in The Human Protein Atlas(https://www. proteinatlas.org/).

\section{GO and KEGG Pathway Enrichment Analysis}

Pathway enrichment analysis was performed on DAVID (https://david.ncifcrf.gov/).Biological significance of differentially expressed genes was explored by GO enrichment analysis including biological process, cellular component and molecular function. KEGG pathway enrichment analysis of differentially expressed genes was performed to explore the critical pathways closely related to C3 up-regulated malignant progression of glioma. We used the "ggplot2" package and "pathview" package (version 1.24.0), which were based on R software to do the visualization of the GO and KEGG signal pathway.

\section{Statistical analysis}

The data were analyzed using GraphPad Prism (version 8.0) and R software (version 4.0). Low and high C3 expression groups were established based on the median C3 mRNA expression value in datasets. The relationship between $\mathrm{C} 3$ expression and a series of categorical variables were analyzed by t-test or Fisher exact-tests. Moreover, we employed a multivariate Cox regression model to probe whether C3 expression was an independent prognostic indicator in glioma patients. Kaplan-Meier curves were utilized to evaluate the prognostic significance of C3. P-values less than 0.05 on both sides were statistically significant.

\section{Results}

\section{Complement C3 Plays an Important Role in the Malignant Progression of Glioma}

In the ONCOMINE database, the complement C3 is highly expressed in most tumors (Figure $1 \mathrm{~A}$ ). We use GEPIA to analyze the mean expression levels of complement C3 in tumor tissue and paired normal tissue, and find that complement C3 is high in glioma (Figure 1B). To explore the prognostic significance of complement $\mathrm{C} 3$ in glioma, we analyze the TCGA-GBMLGG database and found that C3 high expression can promote the malignant progression of glioma $(P<0.001)$ (Figure $1 C)$. The same conclusion is verified in CGGA, Rembrandt and Gravendeel database (Figure $1 \mathrm{E}-\mathrm{G}$ ). We use a multivariate $\mathrm{COX}$ regression model to analyze detailed associations between $\mathrm{C} 3$ expression and clinical features. As shown in (Table 1), the expression of complement C3 is an independent factor to affect patient survival.

\section{Complement C3 Causes Malignant Progression of Glioma Through Nf-Kb and Jak-Stat Signaling Pathway}

In the TCGA-GBMLGG database, C3 high group and low group do difference analysis, to obtain 1501-regulated genes and 731 down-regulated genes, based on the cut-off criteria $(P<0.05$ and fold change $\geq 2$ ) (Figure $2 A)$. The up-regulated genes in the top 200 p-values are selected for GO biological function enrichment analysis. In terms of biological process, C3 up-regulated genes are significantly enriched in the inflammatory reaction and immune response.For cellular components, C3 up-regulated genes are significantly enriched in cell membranes and extracellular matrices. Regarding the molecular function, the up-regulation genes are significantly enriched in cytokine receptor activation (Figure 2B). These significant enrichments can help us further understand the role of $\mathrm{C} 3$ in glioma occurrence and progress. Furthermore, KEGG pathway enrichment analysis shows that the $C 3$ up-regulation genes are associated with NF-kB and JAK-STAT signaling pathways (Figure 2C). Similarly, GSEA also reflects that the C3 high expression group up-regulates NF-kB and JAK-STAT signaling pathways (Figure 2D). The KEGG analysis reveals that C3 up-regulated genes increase the expression of the relevant genes of these two signaling pathways, thereby promoting the malignant progression of gliomas (Figure 2E-F). Gene co-expression

Citation: ChunlanNing\#, YongchangYang\#, YangXie, YanchengLiu, YidingAi, XingXu, XianglianGe, TailongYi, ShengfuShen, Xun Jin*.Complement C3 Triggers Malignant Progression of Mesenchymal Subtype Glioma

Op Acc J Bio Sci \& Res 9(1)-2021 
network is constructed to detect genes showing similar trends (Figure 2G).

\section{Complement C3 Specifically Promotes the Growth of Mesenchymal Subtype Gliomas and Trigger Chemoradiotherapy Resistance}

Immunohistochemical staining reveals that C3 is highly expressed in high-grade glioma, especially in area of necrosis (Figure 3A). In the Rembrandt database, C3 mRNA is highly expressed in MES subtype glioma (Figure 3B). C3 high expression and MES subtype correlation signaling pathways are significantly enriched $(\mathrm{P}<0.01)$ (Figure $3 \mathrm{C})$. In fact, MES subtype gliomas are highly radiotherapy and chemotherapy resistant. Therefore, we analyze the effect of different treatments on the prognosis of glioma. We found that chemoradiotherapy can significantly prolong the survival of glioma patients (Figure 3D). However, in the group of chemoradiotherapy, C3 high expression might cause chemoradiotherapy resistance in glioma patients and affect their prognosis (Figure 3E).

\section{Discussion}

Gliomas account for approximately $80 \%$ of primary central nervous system (CNS) malignant tumors, with high invasiveness, recurrence, high mortality and other characteristics [2]. Currently, the standard treatment for glioma is surgery combined with radiotherapy and chemotherapy $[22,23]$. In fact, due to the deep location of glioma and chemoradiotherapy resistance, the prognosis of patients remains poor $[24,25]$. Therefore, in response to these difficulties, we need to propose new treatment options.

Studies have shown that patients with MES signatures belong to the poor prognosis subtype and are resistant to standard treatments $[26,27]$. In this study, we analyzed that C3 high expression causes chemoradiotherapy resistance in MES subtype gliomas. We also found that C3 high expression promotes chemoradiotherapy resistance in gliomas through NF-kB and JAK-STAT signaling pathways. Similarly, a previous study found that a subset of the PN GSCS undergoes differentiation to a MES state [28] in a TNF-a/ NF-kB-dependent manner with an associated enrichment of CD44 subpopulations and radioresistant phenotypes $[29,30]$. They further show that the MES signature, CD44 expression, and NF-kB activation correlate with lower radiation response and shorter survival in patients [30].

In summary, the expression of C3 is an important factor affecting the chemoradiotherapy resistance of gliomas. Our data shows that C3 high expression may activate NF-kB and JAK-STAT signaling pathway to promote the chemoradiotherapy resistance of glioma, leading to poor prognosis. Therefore, it is a new therapeutic scheme for targeting $\mathrm{C} 3$ and associated signaling pathways to inhibit the chemoradiotherapy resistance of gliomas and improve the prognosis of patients.

\section{Funding}

Science and Technology Support Plan Key Projects of Tianjin (grant Nos.18YFZCSY00690,20YFZCSY00070,), National Natural Science Foundation of China (grant Nos.82073276) and Scientific Research Foundation of Tianjin Medical University Cancer Institute and Hospital (grant Nos. B1807).

\section{Conflicts of Interest}

The authors declare that they have no known competing financial interests or personal relationships that could have appeared to influence the work reported in this paper.

\section{Availability of Data and Material}

Not applicable.

Code Availability

Not applicable.

\section{Ethics Approval}

Not applicable.

\section{Authors' Contributions}

1. Conception and design: C Ning,Y Yang;

2. Collection and assembly of data: C Ning;

3. Data analysis and interpretation: Y Yang;

4. Manuscript writing: All authors;

5. Final approval of manuscript: All authors.

\section{References}

1. Louis DN (2016) The 2016 World Health Organization Classification of Tumors of the Central Nervous System: a summary. Acta Neuropathol 131(6): 803-820.

2. Shergalis A (2018) Current Challenges and Opportunities in Treating Glioblastoma. Pharmacol Rev 70(3): 412-445.

3. Park EC (2013) Differential expression of MicroRNAs in patients with glioblastoma after concomitant chemoradiotherapy. OMICS 17(5): 259268.

4. Tan AC (2020) Management of glioblastoma: State of the art and future directions. CA Cancer J Clin 70(4): 299-312.

5. Chen R (2017) Glioma Subclassifications and Their Clinical Significance. Neurotherapeutics 14(2): 284-297.

6. Jeanmougin M (2020) Improved prognostication of glioblastoma beyond molecular subtyping by transcriptional profiling of the tumor microenvironment. Mol Oncol 14(5): 1016-1027.

7. Wu W (2021) Glioblastoma multiforme (GBM): An overview of current therapies and mechanisms of resistance. Pharmacol Res 171: 105780.

8. Jin X (2017) Targeting glioma stem cells through combined BMI1 and EZH2 inhibition. Nat Med 23(11): 1352-1361.

9. van den Bent (2017) Interim results from the CATNON trial (EORTC study 26053-22054) of treatment with concurrent and adjuvant 
temozolomide for $1 \mathrm{p} / 19 \mathrm{q}$ non-co-deleted anaplastic glioma: a phase 3, randomised, open-label intergroup study. Lancet 390(10103): 16451653.

10. Stupp, R (2005) Radiotherapy plus concomitant and adjuvant temozolomide for glioblastoma. N Engl J Med 352(10): 987-996.

11. Shalapour S, Karin M (2019) Pas de Deux: Control of Anti-tumor Immunity by Cancer-Associated Inflammation. Immunity 51(1): 15-26.

12. McKelvey KJ (2018) Radiation, inflammation and the immune response in cancer. Mamm Genome 29(11-12): 843-865.

13. Reis ES (2019) New insights into the immune functions of complement. Nat Rev Immunol 19(8): 503-516.

14. Morgan BP (2015) The role of complement in neurological and neuropsychiatric diseases. Expert Rev Clin Immunol 11(10): 1109-1119.

15. Orsini F (2014) Versatility of the complement system in neuroinflammation, neurodegeneration and brain homeostasis. Front Cell Neurosci 8: 380.

16. Sekar A (2016) Schizophrenia risk from complex variation of complement component 4. Nature 530(7589): 177-83.

17. Farina C, Aloisi F, Meinl E (2007) Astrocytes are active players in cerebral innate immunity. Trends Immunol 28(3): 138-145.

18. Komotar RJ (2008) The role of complement in stroke therapy. Adv Exp Med Biol 632: 23-33.

19. Singhrao SK (1999) Increased complement biosynthesis by microglia and complement activation on neurons in Huntington's disease. Exp Neurol 159(2): 362-376.

20. Hu WT (2016) CSF complement 3 and factor $\mathrm{H}$ are staging biomarkers in Alzheimer's disease. Acta Neuropathol Commun 4: 14.

21. Boire A (2017) Complement Component 3 Adapts the Cerebrospinal Fluid for Leptomeningeal Metastasis. Cell 168(6): 1101-1113 e13.

22. Stupp R (2007) Chemoradiotherapy in malignant glioma: standard of care and future directions. J Clin Oncol 25(26): 4127-4136.

23. Li G (2020) Gene signatures based on therapy responsiveness provide guidance for combined radiotherapy and chemotherapy for lower grade glioma. J Cell Mol Med 24(8): 4726-4735.

24. Phillips HS (2006) Molecular subclasses of high-grade glioma predict prognosis, delineate a pattern of disease progression, and resemble stages in neurogenesis. Cancer Cell 9(3): 157-173.

25. Oldrini B (2020) MGMT genomic rearrangements contribute to chemotherapy resistance in gliomas. Nat Commun 11(1): 3883.

26. Bhat KPL (2013) Mesenchymal differentiation mediated by NF-kappaB promotes radiation resistance in glioblastoma. Cancer Cell 24(3): 331346.

27. Colman H (2010) A multigene predictor of outcome in glioblastoma. Neuro Oncol 12(1): 49-57.

28. Nakano I (2015) Stem cell signature in glioblastoma: therapeutic development for a moving target. J Neurosurg 122(2): 324-30.

29. Zhang Z (2021) Identification of a mesenchymal-related signature associated with clinical prognosis in glioma. Aging (Albany NY) 13(9): 12431-12455.

30. Magne N (2006) NF-kappaB modulation and ionizing radiation: mechanisms and future directions for cancer treatment. Cancer Lett 231(2): 158-68.

*Corresponding author: Xun Jin, China, Email: jinx2354@163.com

Next Submission with BGSR follows:

- Rapid Peer Review

- Reprints for Original Copy

- E-Prints Availability

- Below URL for auxiliary Submission Link: https://biogenericpublishers.com/submit-manuscript/

Citation: ChunlanNing\#, YongchangYang\#, YangXie, YanchengLiu, YidingAi, XingXu, XianglianGe, TailongYi, ShengfuShen, Xun Jin*.Complement C3 Triggers Malignant Progression of Mesenchymal Subtype Glioma 